\title{
Mechanistic added value of a trans- Sulfonamide-Platinum-Complex in human melanoma cell lines and synergism with cis-Platin
}

Alba Agudo-López, Elena Prieto-García', José Alemán², Carlos Pérez', C. Vanesa Díaz-García', Lucía Parrilla-Rubio³, Silvia Cabrera ${ }^{4}$, Carmen Navarro-Ranninger ${ }^{4}$, Hernán Cortés-Funes ${ }^{1,3}$, José A. López-Martín ${ }^{1,3+}$

and M. Teresa Agulló-Ortuño ${ }^{1 *+}$

\begin{abstract}
Background: Cisplatin is a potent antitumor agent. However, toxicity and primary and secondary resistance are major limitations of cisplatin-based chemotherapy, leading to therapeutic failure. We have previously reported that mono-sulfonamide platinum complexes have good antitumor activity against different tumoral cell lines and with a different and better cytotoxic profile than cisplatin. Besides, $\mathrm{N}$-sulfonamides have been used extensively in medicinal chemistry as bactericides, anticonvulsant, inhibitors of the carbonic anhydrase, inhibitors of histone deacetylases, and inhibitors of microtubule polymerization, among others.

Methods: We aimed to compare the cytotoxic effects of cisplatin and a trans-sulfonamide-platinum-complex (TSPC), in two human melanoma cell lines that differ in their TP53 status: SK-MEL-5, TP53 wild type, and SK-MEL-28, TP53 mutated. We performed cytotoxicity assays with both drugs, alone and in combination, cell cycle analyses, western blotting and immunoprecipitation, and fluorescence immunocytochemistry.

Results: TSPC had similar antiproliferative activity than cisplatin against SK-MEL-5 (3.24 \pm 1.08 vs $2.89 \pm 1.12 \mu \mathrm{M})$ and higher against SK-MEL-28 cells (5.83 \pm 1.06 vs $10.17 \pm 1.29 \mu \mathrm{M}$ ). Combination of both drugs inhibited proliferation in both cell lines, being especially important in SK-MEL-28, and showing a synergistic effect. In contrast to cisplatin, TSPC caused G1 instead G2/M arrest in both cell lines. Our present findings indicate that the G1 arrest is associated with the induction of CDKN1A and CDKN1B proteins, and that this response is also present in melanoma cells containing TP53 mutated. Also, strong accumulation of CDKN1A and CDKN1B in cells nuclei was seen upon TSPC treatment in both cell lines.
\end{abstract}

Conclusions: Overall, these findings provide a new promising TSPC compound with in vitro antitumor activity against melanoma cell lines, and with a different mechanism of action from that of cisplatin. Besides, TSPC synergism with cisplatin facilitates its potential use for co-treatment to reduce toxicity and resistance against cisplatin. TSPC remains

a promising lead compound for the generation of novel antineoplastic agent and to explore its synergism with other DNA damaging agents.

Keywords: Cisplatin, Transplatin, Mono-Sulfonamide, Melanoma, Cell Cycle control, Mechanisms of action

\footnotetext{
* Correspondence: agullo@h12o.es

${ }^{\dagger}$ Equal contributors

'Laboratory of Translational Oncology, Instituto de Investigación Sanitaria

Hospital 12 de Octubre (i + 12), Avda de Córdoba S/N, 28041 Madrid, Spain

Full list of author information is available at the end of the article
} 


\section{Background}

Cisplatin (cis-diamminedichloroplatinum(II) or CDDP) is one of the most successful traditional antitumoral metal compounds used in oncology. Its mode of action is mediated by its interaction with DNA to form DNA adducts, which activate several signal transduction pathways leading to cell death $[1,2]$. However, it is associated with different adverse side effects, such as dose-limiting nephrotoxicity, peripheral neuropathy, electrolyte disturbance, tinnitus and hearing loss [3]. In addition, primary or secondary resistance to CDDP may occur, leading to therapeutic failure $[2,4]$. Many platinum compounds have been designed with the aim to broaden the spectrum of activity, reduce side effects, and overcome resistance to CDDP [5-8].

On the other hand, $N$-sulfonamides have been used extensively in medicinal chemistry as inhibitors of histone deacetylases and microtubule polymerization, among other targets [9-11]. Besides the large number of publications concerning to the use of sulfonamides, the synthesis of platinum compounds containing sulfonamides in their structure has been scarce. The most promising results are given by those compounds which activate alternative signaling pathways $[12,13]$. Therefore, research on new metallodrugs remains an area of interest, especially those with different mechanisms of action from CDDP.

Recently, we published the synthesis and evaluation of a series of trans-sulfonamide platinum complexes showing antitumor activity [14]. Among these compounds, trans-Dichlorido [(rac)-2-(5-(dimethylamino)naphthalene-1-sulfonamido)cyclohexylamino] (dimethylsulfoxide)platinum(II) (hereinafter TSPC), (Fig. 1) demonstrated similar or superior activity than CDDP against a panel of tumor cell lines, including melanoma. Melanoma is a particularly aggressive cancer that has poor prognosis due to resistance to multiple chemotherapy regimens, including in most cases CDDP $[15,16]$. This highlights the urgency of implementing treatment

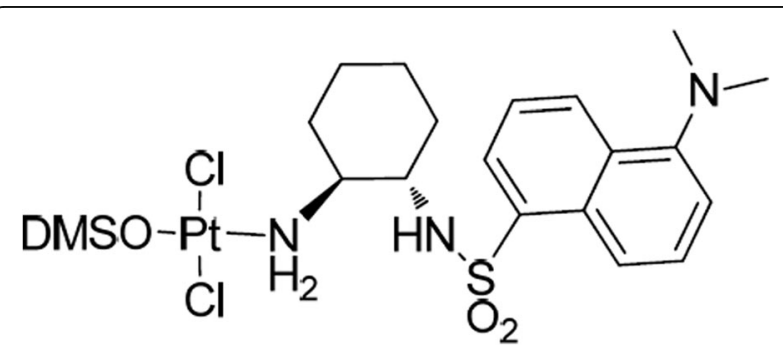

Fig. 1 trans-Dichlorido [(rac)-2-(5-(dimethylamino)naphthalene-1sulfonamido)cyclohexylamino] (dimethylsulfoxide)platinum(II) compound (TSPC). Chemical Formula: $\mathrm{C}_{20} \mathrm{H}_{31} \mathrm{Cl}_{2} \mathrm{~N}_{3} \mathrm{O}_{3} \mathrm{PtS}$. Molecular Weight: $691.5924 \mathrm{~g} / \mathrm{mol}$ strategies for melanoma with more effective and less toxic novel drugs.

Therefore, we decided to study several mechanistics aspects of TSPC in two melanoma cell lines with a different TP53 status: SK-MEL-5 (TP53 wild-type) and SK-MEL-28 (TP53 mutated) [17]. TP53 is a tumor suppressor protein that facilitates antitumor drug response using a variety of key cellular functions, including cell cycle arrest, senescence, and apoptosis [18], whose role in the mode of action of CDDP has been extensively described [1]. These functions usually cease once TP53 mutates, as occurs in nearly $50 \%$ of cancers, and some TP53 mutants even exhibit gain-of-function effects, which can lead to even greater drug resistance [18]. It is therefore important to test the effectiveness of new drugs in TP53 mutants.

In this study, we show the in vitro antitumor activity of TSPC and CDDP in these melanoma cell lines and mechanistic differences in cell cycle effects. We also aim to explore if these differential effects might be used as a basis for a synergism between both compounds.

\section{Methods \\ Cell lines and reagents}

Human melanoma cell lines SK-MEL-5 and SK-MEL-28 were obtained from American Type Culture Collection (ATCC, Manassas, VA) in February 2010. Low passage cells were cultured in RPMI 1640 medium supplemented with $10 \%$ fetal bovine serum, $100 \mathrm{U} / \mathrm{mL}$ penicillin, $100 \mu \mathrm{g} / \mathrm{mL}$ streptomycin and $2 \mathrm{mM} \mathrm{L}$-glutamine, under standard culture conditions $\left(37{ }^{\circ} \mathrm{C}, 95 \%\right.$ humidified air and 5\% $\mathrm{CO}_{2}$ ). RPMI 1640 and other culture materials were from Lonza Ltd. (Verviers, BEL). TSPC was synthesized and characterized in the Inorganic Chemistry Department of the Universidad Autónoma de Madrid (Spain), as previously reported [14]. CDDP was obtained from Selleck Chemicals LLC (Houston, TX). Drugs were dissolved in dimethyl sulfoxide (DMSO) at $100 \mathrm{mM}$ of stock solution, and stored at $-20{ }^{\circ} \mathrm{C}$ until use.

\section{Proliferation assays}

Briefly, cells were seeded in a 96-well flat-bottom plate at 5000 cells/well and cultured for $24 \mathrm{~h}$ prior to exposure to CDDP or TSPC at varying concentrations, from 0 to $100 \mu \mathrm{M}$ for $72 \mathrm{~h}$. For combination assays, cells were treated with CDDP ranging from 0.5 to $10 \mu \mathrm{M}$ and two different doses of TSPC (1 and $5 \mu \mathrm{M})$. Results were expressed as a percentage relative to vehicle-treated control (0.1\% DMSO was added to untreated cells). Viability was determined using the WST-1 method (Roche, Mannheim) following the manufacter's instructions. The $50 \%$ inhibitory concentration $\left(\mathrm{IC}_{50}\right)$ was calculated by nonlinear regression fit of the mean values of the data 
obtained in at least three independent experiments using GraphPad Prism software version 5.0 (San Diego, CA).

The type of drug interaction was analysed by the Chou-Talalay method [19]. This method provides a combination index $(\mathrm{CI})$ that allows quantitative determination of drug interactions, where $\mathrm{CI}<1,1$, and $>1$ indicate synergism, additive effect or antagonism, respectively, considering synergism as more than additive effect and antagonism as less than additive effect. This method also provides a dose reduction index (DRI) that measure how many-fold the dose of a drug can be reduced in combination with respect to the drug alone. The CI and the DRI were calculated using the CompuSyn software (Comb ComboSyn Inc, Paramus, NJ).

\section{Cell cycle and apoptosis analysis}

Cells were treated for $24 \mathrm{~h}$ with equimolar concentrations of either CDDP or TSPC. The cell cycle progression was examined by flow cytometry after staining with propidium iodide (PI). DNA content and cell cycle analyses were performed by using a FACScalibur flow cytometer and the CellQuest software (Becton Dickinson Biosciences). Apoptosis was studied with the APOBrdUTunel assay kit (Life Technologies Inc. Gaithersburg, MD) following the experimental protocol provided by the manufacturer, and analyzed with a FACScalibur flow cytometer and the CellQuest software (BD Biosciences). All experiments were performed in triplicate.

\section{Western blot and immunoprecipitation assays}

Cells untreated (control) or treated with equimolar concentrations of either CDDP or TSPC for 24 h were lysed with MCL1 lysis buffer in the presence of protease and phosphatase cocktail inhibitor (Sigma-Aldrich Co. LLC, St Louis, MO) following the manufacter's protocol. Total protein concentrations were determined using the $\mathrm{BCA}$ protein assay kit (Thermo Scientific Meridian Rd, Rockford, IL). Protein lysates $(30 \mu \mathrm{g})$ were subjected to SDS-PAGE on $15 \%$ polyacrylamide gel. The separated proteins were transferred on to PVDF membrane followed by blocking with $5 \%$ non-fat milk powder $(\mathrm{w} / \mathrm{v})$ in TTBS (10 mM Tris, $100 \mathrm{mM} \mathrm{NaCl}, 0.1 \%$ Tween 20). Membranes were probed for the protein levels of TP53, CDKN1A (p21 $\left.{ }^{\mathrm{Cip} 1}\right), \quad \mathrm{CDKN} 1 \mathrm{~B} \quad\left(\mathrm{p} 27^{\mathrm{Kip} 1}\right), \quad \mathrm{CDKN} 2 \mathrm{~B}$ $\left(\mathrm{p} 15^{\mathrm{INK} 4 \mathrm{~b}}\right), \mathrm{CDK} 2, \mathrm{CDK} 4, \mathrm{CCND} 1$ (Cyclin D1), CCNE2 (Cyclin E2), phospho-CDK1 (P-cdc2) and GAPDH using specific primary antibodies (Cell Signaling Tech Inc, Danvers, MA) diluted $1 / 1000$ in blocking solution followed by peroxidase-conjugated appropriate secondary antibody (Santa Cruz Biotechnology Inc, Dallas, TX), and visualized by Immun-Star WesternC kit (Bio-Rad) detection system. Results were scanned with Image Quant LAS 4000 Imaging densitometer and quantify with Image Quant TL software (GE healthcare, Life
Sciences). To ensure that control cells gave reproducible baseline protein levels, we carefully maintained cells cultures in a constant semiconfluent and logarithmically growing state throughout each experiment.

Pierce Classic IP Kit (Thermo Scientific) was used for immunoprecipitation assays, and manufacturer's instructions were followed. Thereby, $1 \mathrm{mg}$ of protein was immunoprecipitated using CDK2 primary antibody (Cell Signaling Tech Inc, Danvers, MA). The immune complex eluted was subjected to SDS-PAGE and transblotted into PVDF membranes as described above. Membranes were probed for the protein levels of CDK2, CDKN1A and CDKN1B.

\section{Fluorescence immunocytochemistry}

Cells were cultured in coverslip glasses for $24 \mathrm{~h}$ with equimolar concentration of either CDDP or TSPC. Untreated cells were used as control. After exposure to the drugs, cells were fixed with $4 \%$ formaldehyde and permeabilized with PBS/triton (0.1\%). For immunofluorescence labeling, cells were incubated with anti-human CDKN1A (1:1000) or CDKN1B (1:1000) monoclonal antibodies (Cell Signaling Tech Inc, Danvers, MA) followed by a secondary antibody conjugated to Alexa Fluor 488 (Life Technologies Inc; Gaithersburg, MD). Nuclei were stained with 2-(4amidinophenyl)-1H-indole-6-carboxamidine (DAPI) (Life Technologies Inc. Gaithersburg, MD). Images from six to ten fields per sample of at least three independent experiments were taken with a LSM 510 Meta Confocal Microscope (Zeiss, Germany).

\section{Recovery experiments}

To study the effects of duration of platinum exposure on cell proliferation, cells were treated with CDDP or TSPC at their corresponding $\mathrm{IC}_{50}$ for $72 \mathrm{~h}$, or with CDDP $1 \mu \mathrm{M}$ (SK-MEL-5) or $3 \mu \mathrm{M}$ (SK-MEL-28) and TSPC 1 or $5 \mu \mathrm{M}$ in combination assays. After this time, cells were washed with PBS and put in drug-free medium to recover. Viable cell number was assessed with Trypan blue and a Neubauer chamber for 7 days of recovery.

\section{Statistical analysis}

Results are expressed as the mean $\pm \mathrm{SD}$, and data are representative of at least three different experiments. Analysis of variance (ANOVA) one way, followed by Dunnett's $t$-test was used to examine differences between groups. For recovery experiments, cell growth was fit to a Gompertz function, typical of cell growth and characterized by a lag period, an exponential and a saturation phase. All the statistical tests were conducted at the two-side 0.05 level of significance. 


\section{Results}

TSPC inhibits growth and induces G1 arrest in human melanoma SK-MEL-5 and SK-MEL-28 cells

Based on our promising previous work with TSPC [14] we decided to study its effect in two melanoma cell lines: SK-MEL-5 (TP53 wild-type) and SK-MEL-28 (TP53 mutated). First, we analysed TSPC antiproliferative activity. As shown in Fig. 2a, TSPC treatment inhibited the growth of human melanoma SK-MEL-5 and SK-MEL-28 cells in a dose-dependent manner. The $\mathrm{IC}_{50}$ of this compound $(3.24 \pm 1.08 \mu \mathrm{M})$ was similar to the one obtained with CDDP $(2.89 \pm 1.12 \mu \mathrm{M})$ in SK-MEL-5, and significantly lower $(p<0.05)$ in SK-MEL-28 $(5.83 \pm 1.06 \mu \mathrm{M}$ vs $10.17 \pm 1.29 \mu \mathrm{M})$.

Next, we examined its effect on cell cycle progression and in apoptosis induction. Consistent with the results on cell growth inhibition, TSPC treatment for $24 \mathrm{~h}$ induced a significant $(p<0.05)$ increment of cell number in G1 phase in both melanoma cell lines (Fig. 2b), whereas CDDP treatment induced S arrest, as has been previously described elsewhere [2]. Apoptosis was not observed by TUNEL assay after $24 \mathrm{~h}$ treatment with neither CDDP nor TSPC, which is consistent with the subG1 cell population identified in cell cycle analyses (Figs. 2b and c). These effects were apparent in both cell lines after a 24-h exposure to any of the two compounds.

\section{Effect of TSPC on $\mathrm{G} 1$ cell cycle regulators}

Based on the G1 arrest induced by TSPC in both cell lines, we assessed the effect of this compound on the cell cycle regulatory molecules that play important roles in G1 phase of cell cycle progression. TP53 is the most important tumor suppressor protein associated with the

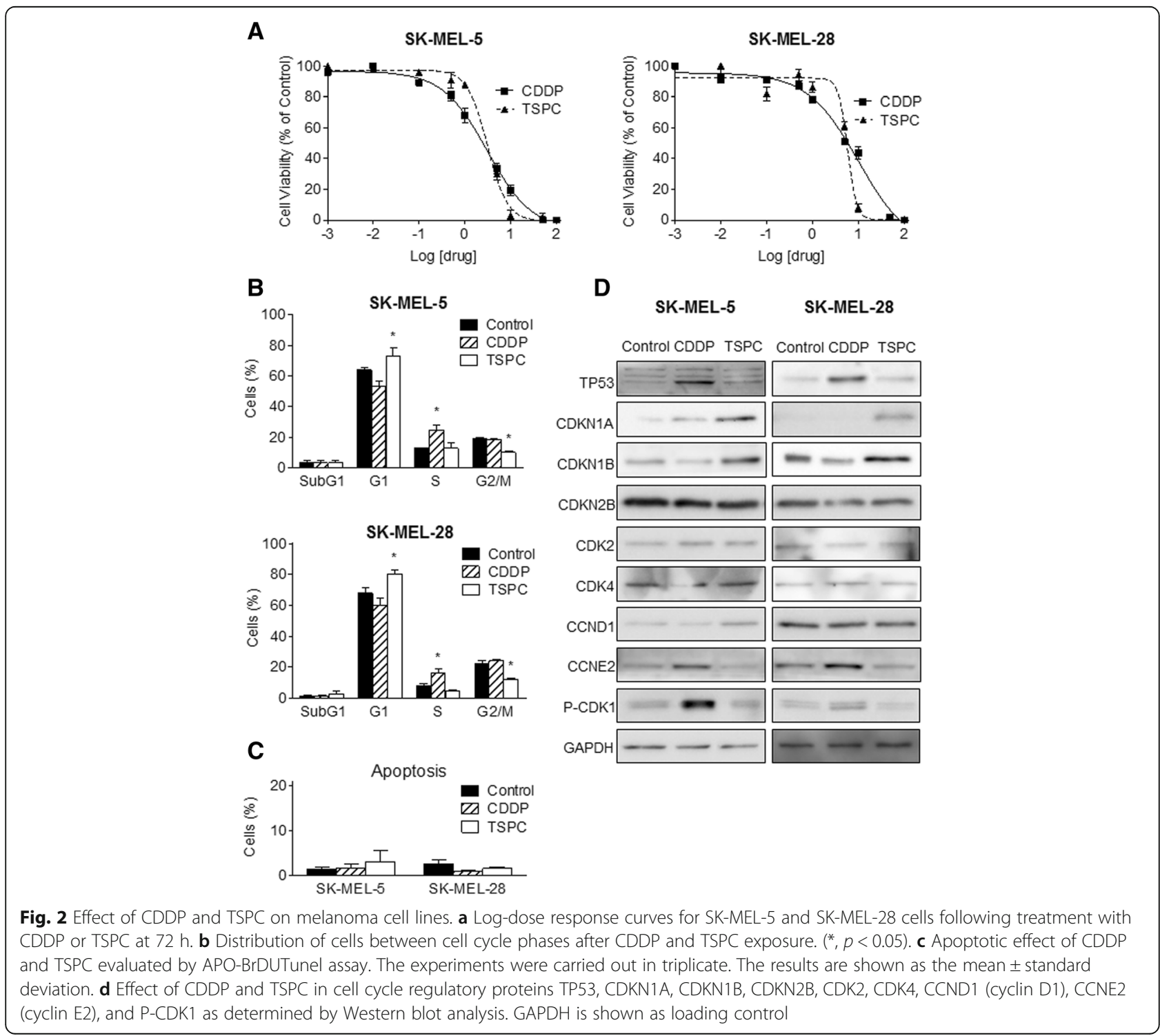


G0-G1 arrest in cell cycle, and a key protein in CDDP mode of action and resistance. Since SK-MEL-28 has a mutation in TP53, we also examined the protein expression of the cyclin-CDK inhibitors (CKIs) CDKN1A (p21 $\left.{ }^{\mathrm{Cip} 1}\right), \mathrm{CDKN} 1 \mathrm{~B}\left(\mathrm{p} 27^{\mathrm{Kip} 1}\right)$, and CDKN2B (p15 $\left.{ }^{\text {INK4b }}\right)$.

Western blot analysis showed that TSPC treatment strongly increased the protein levels of CDKN1A and CDKN1B and had no effect on TP53 levels in both cell lines (Fig. 2d). The observed strong induction in CDKN1A and CDKN1B protein levels by TSPC was not due to an overall change in protein levels as confirmed by probing the same membranes with GAPDH antibody (Fig. 2d). Thus, treatment of SK-MEL-5 and SK-MEL-28 cells with TSPC caused an increased in CDKN1A and CDKN1B expression, with correlated with the G1 arrest at $24 \mathrm{~h}$. Conversely, CDDP increased the protein levels of TP53 in both cell lines accompanied by an increase in CDKN1A in SK-MEL-5 but not in SK-MEL-28. CDDP also slightly decreased the protein levels of CDKN1B in both cell lines. No differences were observed in CDKN2B levels with any treatment.

We also assessed the effect of TSPC on the protein levels of CDKs and cyclins involved in G1 phase and G1 to $S$ phase transition of cell cycle. As shown in Fig. 2d, drug treatments did not show any detectable change in the protein levels of CDK2 and CDK4, and only a slight increase in cyclin D1 with TSPC in SK-MEL-5 was observed. On the other hand, cyclin E2 levels were strongly increased by CDDP, and conversely reduced by TSPC in both cell lines.

In order to assess the mechanism of action of CDDP in the absence of TP53, we also examined the phosphorylation state of CDK1 (cdc2), which have been shown to be associated with $S$ and G2/M arrest and with CDDP mechanism of action. Western blot analysis showed that CDDP treatment strongly induced the protein levels of phospho-CDK1 in both cell lines, while TSPC had no effect (Fig. 2d). Densitometric analysis of proteins from Fig. 2 d is show in Fig. 3.

\section{TSPC inhibits CDK2-cyclin E via CDKN1A and CDKN1B}

Since up-regulation in CKIs levels was observed, and these proteins exert their inhibitory effect and G1 cell cycle arrest by direct binding to CDK2-cyclin E complexes, we next examined CDK2-CKIs binding by immunoprecipitation assay. As shown Fig. 4, TSPC treatment showed an increased binding of CDKN1A and CDKN1B with CDK2 in both cell lines. CDDP also showed an increased binding of CDKN1A, bigger than that observed with TSPC in SK-MEL-5.

The inhibitory effect of CKIs on CDK-cyclin complexes occurs in the nucleus, so we next examined by immunofluorescence and confocal microscopy the localization of CKIs in this cell lines. As shown in Fig. 5a, both CDKN1A and CDKN1B were mainly localized in the nucleus in untreated cells. TSPC exposure induced an increase of CDKN1A and CDKN1B in the nucleus in SK-MEL-5 and SK-MEL-28, while CDDP treatment seemed to slightly modified CDKN1B levels in both cell lines. No CDKN1A staining was perceivable in SK-MEL28 in control and CDDP-treated cells. Confocal images matched the results obtained by western blot (Fig. 2d).

In addition, we determined the percentage of nuclear CDKN1A and CDKN1B staining in cell lines exposed to CDDP or TSPC. As shown in Fig. 5b, cells treated with TSPC had a higher percentage of nuclear CDKN1A and CDKN1B than cells treated with CDDP (SK-MEL-5: CDKN1A $25.36 \pm 11.53$ vs $5.45 \pm 4.16$; CDKN1B $54.04 \pm$ 10.36 vs $30.70 \pm 8.85$ ) (SK-MEL-28: CDKN1A $15.31 \pm$ 5.28 vs $1.5 \pm 0.63$; CDKN1B $62.11 \pm 10.50$ vs $23.53 \pm$ 8.31). No significant changes of CDKN1A and CDKN1B subcellular location were observed after treatment with CDDP. By contrast, a significant increase in the nuclear staining of both CKIs after exposure to TSPC was seen.

\section{TSPC in combination}

Once analysed the effect of TSPC on cell cycle and cell growth, we next examined how long this effect would last. Cell treated with TSPC recovered a normal proliferation rate after drug removal. However, cell treated with CDDP did not recover (Fig. 6a). Seeing these results, as well as the differential effects in cell cycle, we thought it could be interesting to explore evidences for synergism using both drugs in combination. As shown in Fig. 6b, addition of TSPC to CDDP increased cell death in a dose dependent manner in both cell lines, being especially important in the case of SK-MEL-28 with TSPC $5 \mu \mathrm{M}$. In fact, the analysis of these drugs interaction by the Chou-Talalay method showed that this increment in cell death was due to a synergistic effect between the drugs $(\mathrm{CI}<1 ; \log \mathrm{CI}<0)$ in SK-MEL-28 at almost every dose and, in SK-MEL-5 at the highest doses of CDDP (Fig. 6c). Due to this synergism, there was a favourable DRI (DRI > 1; $\log$ DRI $>0$ ) in CDDP dose and in some doses of TSPC in SK-MEL-5 and in both CDDP and TSPC in SK-MEL-28 (Fig. 6d).

Taking into consideration this synergism we studied whether cells treated with both drugs in combination were able to recover after drug removal. For this purpose we treated cells with CDDP at a dose lower than their respective $\mathrm{IC}_{50}: 1 \mu \mathrm{M}$ (SK-MEL-5) or $3 \mu \mathrm{M}$ (SK-MEL28 ), and TSPC 1 or $5 \mu \mathrm{M}$. In all the cases cells recovered in the same way (Fig. 6e).

\section{Discussion}

Platinum complexes are widely used in cancer therapy. The successful clinical applications of CDDP have inspired the synthesis and investigation of numerous 


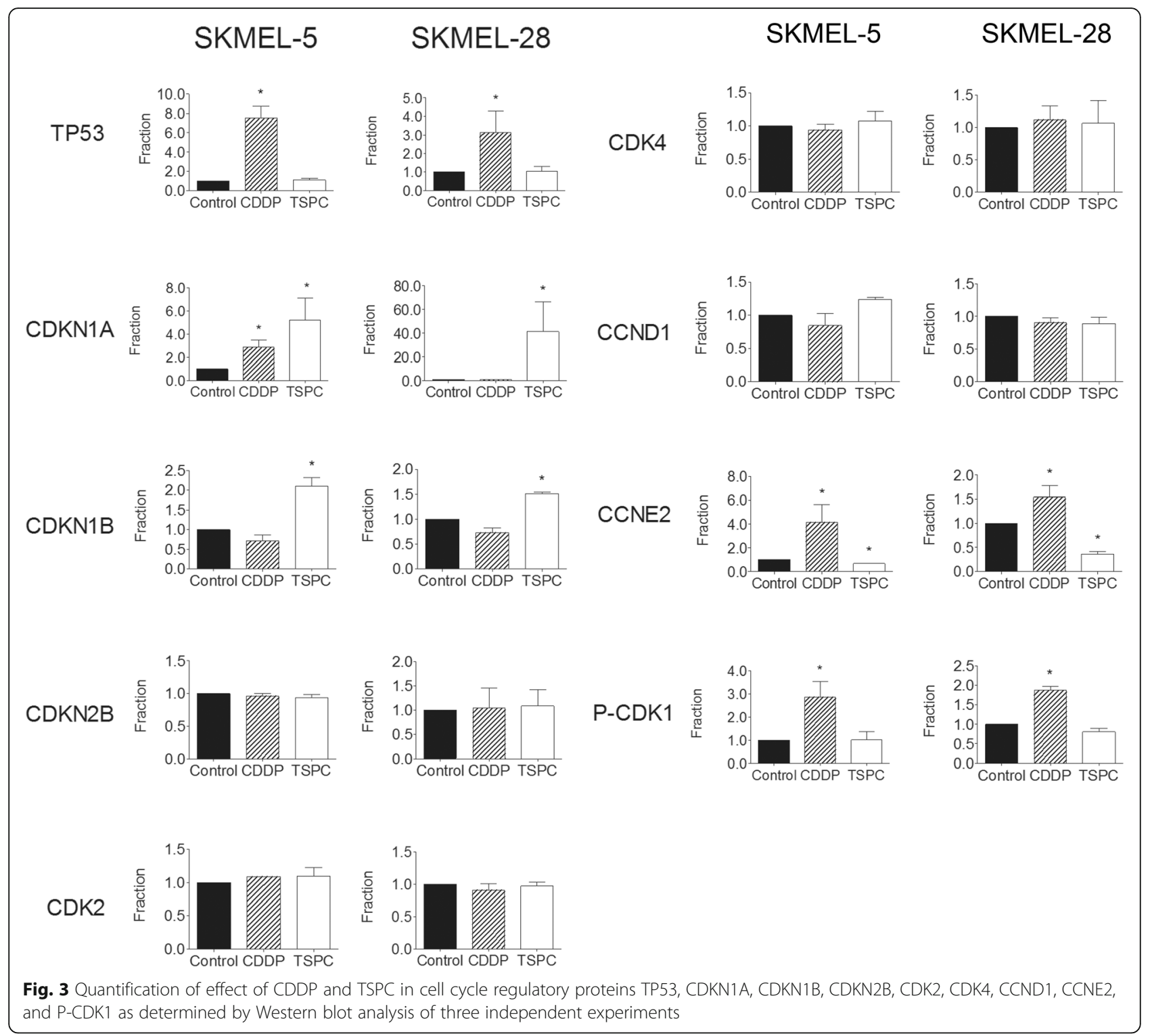

platinum compounds as drug candidates. All the commercially available platinum compounds are based in a cis-isomerism.

In this study, the cellular and molecular effects of the trans-platinum derivative TSPC were investigated and directly compared with those of CDDP in SK-MEL-5 and SK-MEL-28 melanoma cell lines. The choice of these cell lines were made on the basis of TP53 status, and in our previous cytotoxicity results in a panel of different cell lines [14].

TSPC showed to be as active as CDDP in SK-MEL-5 and more active in SK-MEL-28, the TP53 mutant line. TP53 plays a very important role in CDDP cytotoxicity [20], so it is not surprising that a TP53 mutant line show some resistance to this drug $[21,22]$. On the other hand, and according to the results obtained in this work, TSPC mode of action is clearly TP53-independent, as there is no change in this protein levels after TSPC treatment, neither in SK-MEL-5 nor in SK-MEL-28 cell lines.

Upon $24 \mathrm{~h}$ incubation with TSPC, cells were arrested in G1 phase that suggest cell cycle delay for a DNA damage response, DNA repair and/or apoptosis. One goal of this study was to define the cell cycle regulatory pathway responsible for the G1 arrest. The results obtained in these two cell lines with different TP53 status show that TSPC inhibits cell proliferation through cell cycle arrest at G1 phase, likely due to the up-regulation of CDK inhibitors CDKN1A and CDKN1B. Progression through the G1 phase of the cell cycle is controlled by CDK4-cyclin D complexes, while CDK2-cyclin E complexes are required for proper G1/S transition and initiation of $S$ [23]. CDKs are negatively regulated by the 


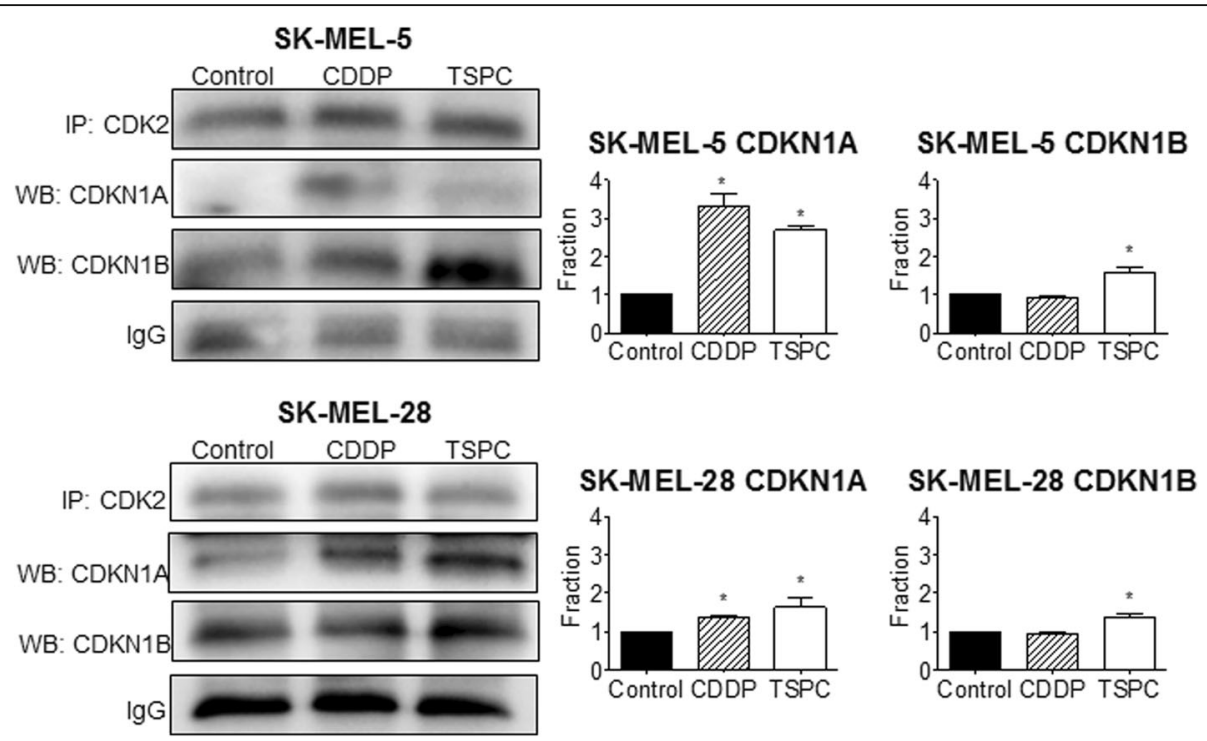

Fig. 4 Co-immunoprecipitation assay of the interaction between CDK2 and CDKN1A, and CDK2 and CDKN1B in SK-MEL-5 and SK-MEL-28 cell lines after CDDP and TSPC exposure. Quantification of CDKN1A and CDKN1B binds to CDK2, detected in co-immunoprecipitated products. ( $\left.{ }^{*}, p<0.05\right)$

binding of CDK inhibitors. INK4 family inhibitors, as CDKN2B, prevent cyclin $\mathrm{D}$ binding to CDK4, while CDKN1A and CDKN1B bind to CDK2-cyclin E complexes and inhibit CDK2 activity [24]. Our results showed that neither CDK4, nor cyclin D1, nor CDKN2B were affected by TSPC in SK-MEL-28 cells. Only a slight increase in cyclin D1 was observed in SK-MEL-5 cells but such levels might be insufficient to induce G1 progression effectively. However, CDKN1A and CDKN1B protein levels were clearly enhanced after TSPC treatment, and cyclin E2 levels were reduced in both cell lines. Besides, co-immunoprecipitation assays showed that both CDKN1A and CDKN1B bind to CDK2 after TSPC treatment. Thus, it can be inferred that TSPC induces G1 arrest in SK-MEL-5 and SK-MEL-28, via CDKN1A and CDKN1B binding to CDK2-cyclin E complexes in a TP53-independent manner (Fig. 7). The cell cycle arrest is a common cellular response to DNA damage and is viewed as a delay period in DNA replication during which the cell can attempt to repair the damage. If this attempt fails, cell death pathways will be activated $[25,26]$. Our present findings indicate that the G1 arrest is associated with an induction of CDKN1A and CDKN1B proteins, and that this response is also seen in melanoma cells containing TP53 mutated.

On the other hand, cells incubated with CDDP were arrested in $\mathrm{S}$ and G2/M phase. Several reports have previously described that CDDP induces S- and G2/M-phase arrests in a sequential manner [27]. These arrests are associated with phosphorylation and proteosomal degradation of $\mathrm{CDC} 25$ phosphatase, with the consequence that CDK within the CDK2/cyclin A and CDK1/cyclin B complexes remain in the inhibitory tyrosine phosphorylated state
[28]. Besides, CDKN1A can bind these CDKs and inhibit these complexes [29], and as an inhibitor of $\mathrm{CDC} 2$ phosphorylation, CDKN1A can be involved in both G1 and G2 cell cycle arrest [30]. Our data from the present study are in line with these previously published studies as CDK1 remain phosphorylated after CDDP treatment, and CDKN1A binds to and inhibit CDK2. However, this setting is different to that of TSPC, where CDC2 remains hypophosphorylated. So, it seems that TSPC has a different mode of action from that of CDDP.

The cell cycle regulatory function of both CDKN1A and CDKN1B is associated with its nuclear localization, but the protein can also localize in the cytoplasm where they could exert different functions [31]. In the nucleus, in addition to the regulation of the cell cycle progression, CDKN1A and CDKN1B are also involved in a variety of transcriptional responses [32]. In the cytoplasm, CDKN1A can initiate antiapoptotic responses by inhibiting proapoptotic kinase ASK1 [33] or by binding to procaspase 3 , thus preventing its proteolytic activation [34]. In a more general sense, it has been suggested that the subcellular localization of CDKN1A defines its function as a tumor suppressor (nuclear localization) or oncoprotein (cytoplasmic localization) [32]. Indeed, some human cancers display elevated levels of cytoplasmic CDKN1A or cytoplasmic CDKN1B, which is associated with poor prognosis [35-38], and badly response to cisplatin based treatment $[39,40]$. In our experiments, the increased nuclear localization of CDKN1A and CDKN1B in both melanoma cell lines after TSPC treatment supports the antitumor activity of this compound.

Recovery experiments showed that while CDDP effect, at least at $\mathrm{IC}_{50}$ dose, was permanent, TSPC treatment 

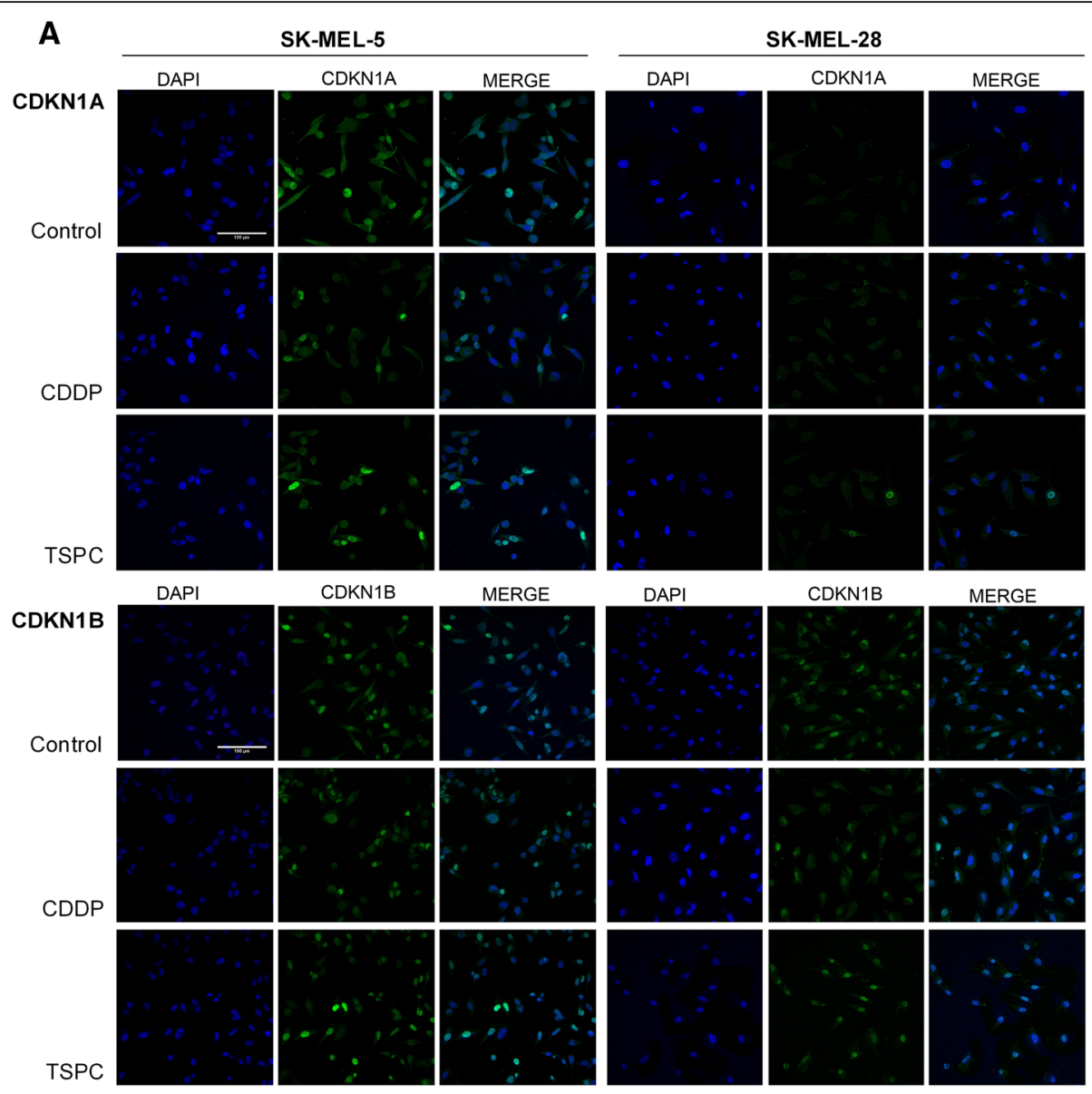

DAPI CDKN1B

MERGE

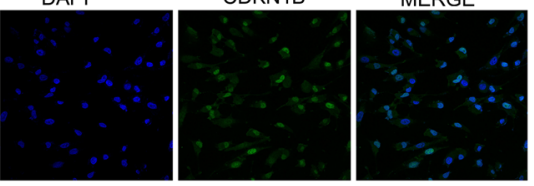

$\mathbf{B}$
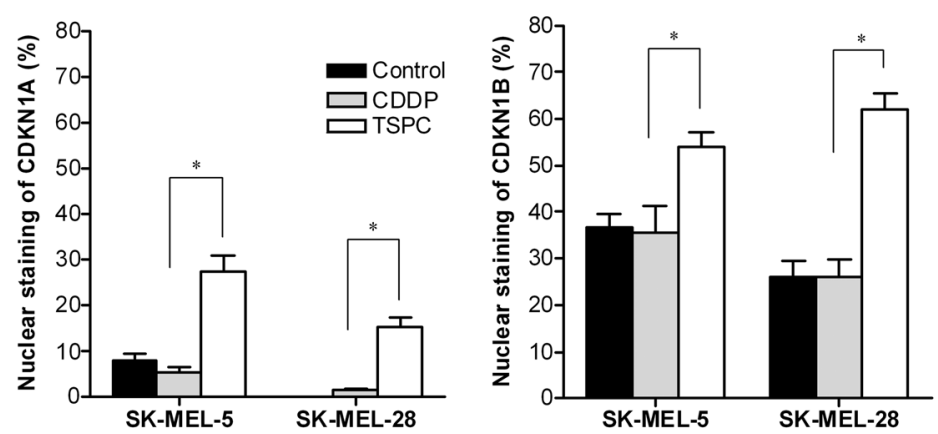

Fig. 5 Cellular distribution of CDKN1A and CDKN1B in melanoma cell lines and its alteration after CDDP or TSPC treatment. a Subcellular distribution of CDKN1A and CDKN1B in SK-MEL-5 and SK-MEL-28 cells, after CDDP and TSPC treatment assessed by confocal microscopy. First and fourth columns: nuclei stained with DAPI; Second and fifth columns: CDKN1A (up) and CDKN1B (down) proteins stained with specific antibody and secondary antibody conjugated with Alexa Fluor 488; Third and sixth columns: colocalization. b Quantitative analysis of nuclear CDKN1A (left) and CDKN1B (right) staining in melanoma cell lines. Staining cells were counted at $200 \times$ magnification from six to ten randomly selected fields. Total 100 cells were counted in each experiment. $\left(^{*}, p<0.05\right)$

was transitory. This is not necessarily a negative result, but it implies that TSPC treatment should be frequent. Thus, in regard to clinical treatment, it would be useful to find an easy and sequentially way of TSPC administration.

CDDP is one of the most potent antitumor agents known. However, toxicity and resistance are major limitations of CDDP-based chemotherapy $[1,2]$. Thus, the potential for use of TSPC in combination with CDDP was explored in terms of eventual synergism. In both cell lines tested, our data show a synergistic effect and a favorable DRI when cells are simultaneously treated with both CDDP and TSPC, which could lead to resistance minimization. This synergy is likely to be due to the mechanistic differences between CDDP and TSPC. 

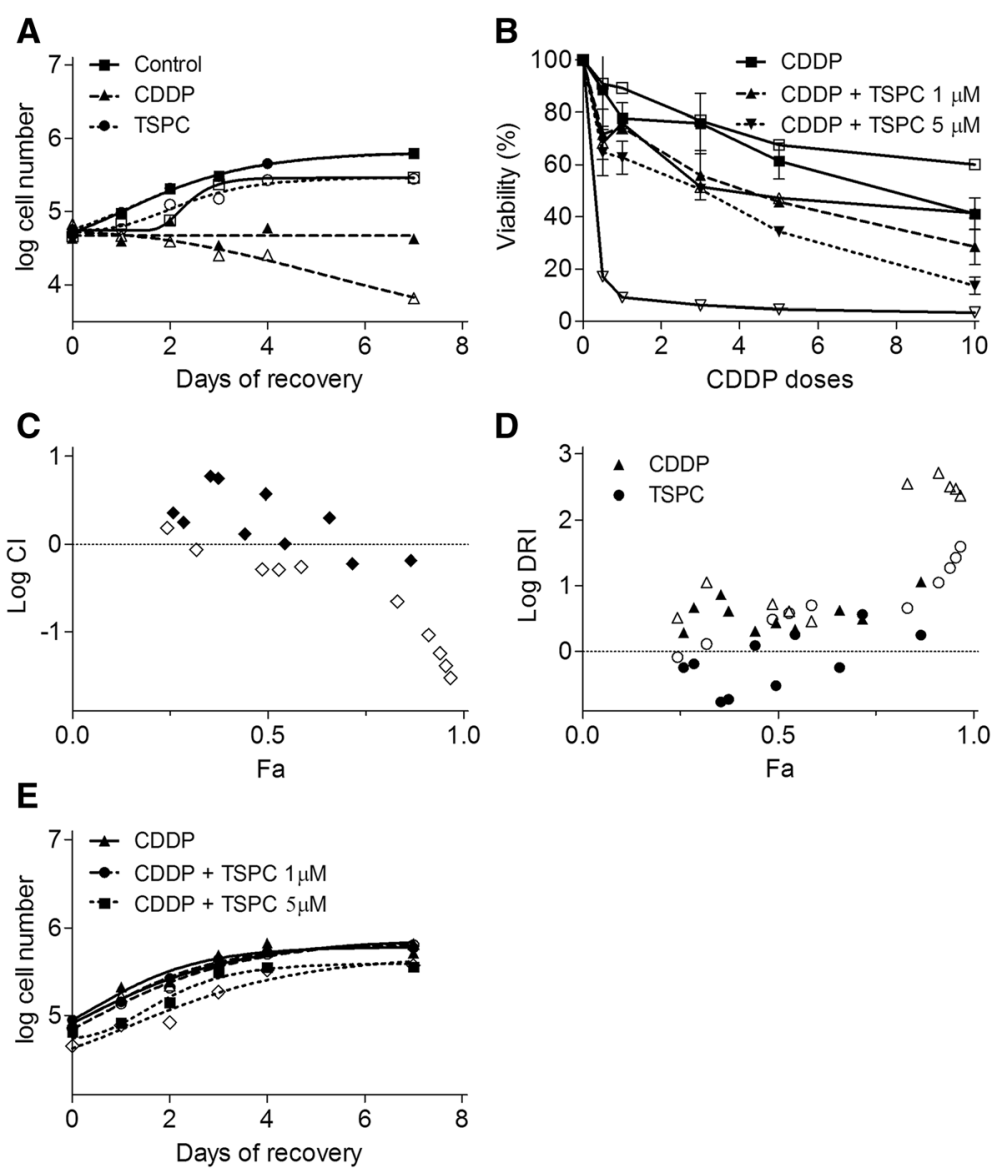

Fig. 6 Effects of CDDP and TSPC combined treatment. a Cell recovery after treatment removal. Cell growth was assessed for 7 days in cells treated with either CDDP (triangles) or TSPC (circles) at their corresponding $I_{50}$ for $72 \mathrm{~h}$ followed by drug removal. Untreated cells were used as control (squares). b Cell viability with drug treatment combination. Viability after treatment with different doses of CDDP ranging from 0.5 to $10 \mu \mathrm{M}$ combined with TSPC $1 \mu \mathrm{M}$ (right side-up triangles) or $5 \mu \mathrm{M}$ (upside-down triangles). Cells just treated with CDDP were used as control (squares). c Fa-log Cl plot. The line represents the additive effect and all the points under it show synergism. d Fa-log DRI plot. The line separates the favourable dose reduction (up) from the unfavourable (down). e Cell recovery after combination treatment removal. Clear shape symbol: SK-MEL-5; Dark shape symbol: SK-MEL-28

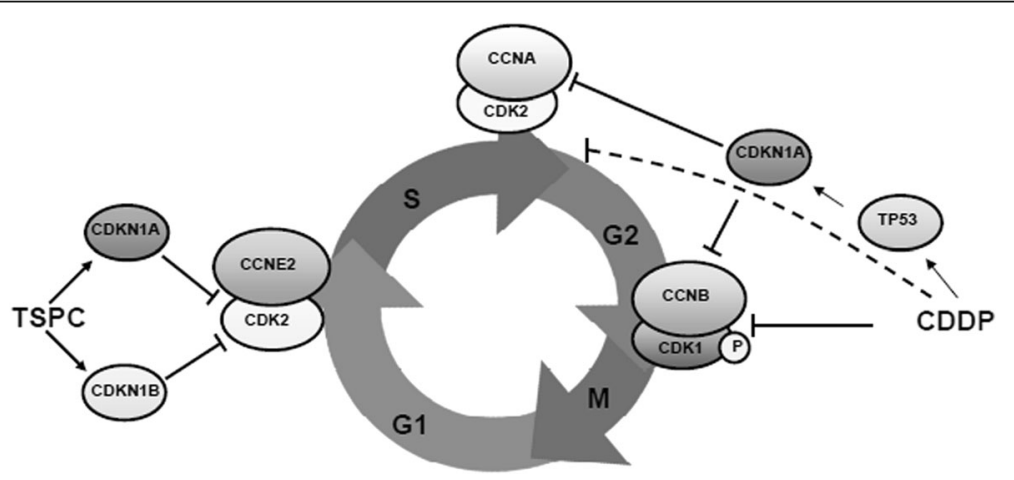

Fig. 7 TSPC induces cell cycle G1 arrest probably mediated by CDKN1A and CDKN1B CKIs in a TP53-independent manner, while CDDP induces S and G2/M arrest via TP53/CDKN1A pathway in SK-MEL-5, and preventing dephosphorylation of CDK1 in both cell lines 


\section{Conclusions}

In this study we present a new promising TSPC compound with in vitro antitumor activity against SK-MEL-5 and SKMEL-28 melanoma cell lines, with a different mechanism of action that of CDDP. From our results it can be inferred that TSPC induces cell cycle G1 arrest probably mediated by CDKN1A and CDKN1B inhibitors in a TP53-independent manner. CDK2-cyclin E complexes are required for G1/S transition. By co-immunoprecipitation assays, we show that CDKN1A and CDKN1B bind to CDK2 after TSPC treatment, inhibiting CDK2 activity. Besides, synergy between TSPC and CDDP facilitates its potential use for co-treatment to reduce toxicity and resistance against CDDP. On the other hand, TSPC remains a promising lead compound for the generation of novel drug candidates with different cytotoxicity profiles from those of CDDP. Moreover, these results could have therapeutic implications for TSPC, because the majority of human solid tumors contain a mutant TP-53 or deleted TP53 gene and are inherently resistant to commonly used DNA-damaging agents.

\section{Abbreviations}

CDDP: Cisplatin or cis-diamminedichloroplatinum(II); Cl: Combination index; CKIs: Cyclin-CDK inhibitors; DRI: Dose reduction index; TSPC: Trans-sulfonamideplatinum-complex

\section{Acknowledgments}

The authors thank F. Bartolomé for assisting with confocal microscopy studies.

\section{Funding}

This work was supported by Fundación Mutua Madrileña (MMA 2013/0069). A.A.-L. received grant support from "Red Temática de Investigación Cooperativa en Cáncer" (ISCiii, Spain). E.P.-G., C.P., and C.V.D.-G., received grant support from Ministerio de Sanidad y Política Social (TRA-151, EC10-228) and from Fundación Mutua Madrileña (MMA 2010/018) (Spain). M.T.A.-O., received grant support from MINECO (Innpacto-2012-1311-300000, Spain). J.A. thanks the Spanish Government for their Ramón y Cajal contract.

\section{Availability of data and materials}

The authors declare that all data generated or analysed during this study are included in this published article.

\section{Authors' contributions}

JA, CN-R, JAL-M and MTA-O design the study. AA-L, EP-G, JA, CP, CVD-G, LP-R, SC, CN-R and, HC-F participated in the data collection and generation of results. JA, AA- L and MTA-O analyzed and interpreted data. AA- $L$ and MTA-O drafted the article which was revised and approved by all authors.

\section{Competing interests}

The authors declare that they have no competing interests.

\section{Consent for publication}

Not applicable.

\section{Ethics approval and consent to participate}

Not applicable.

\footnotetext{
Author details

'Laboratory of Translational Oncology, Instituto de Investigación Sanitaria Hospital 12 de Octubre (i + 12), Avda de Córdoba S/N, 28041 Madrid, Spain. ${ }^{2}$ Organic Chemistry Department (Module 1), Universidad Autónoma de Madrid, C/Fco Tomás y Valiente, 5. Cantoblanco, 28049 Madrid, Spain. ${ }^{3}$ Medical Oncology Service, Hospital Universitario 12 de Octubre, Avda de Córdoba S/N, 28041 Madrid, Spain. ${ }^{4}$ Inorganic Chemistry Department
}

(Module 7), Universidad Autónoma de Madrid, C/Fco Tomás y Valiente, 5, Cantoblanco, 28049 Madrid, Spain.

Received: 14 November 2016 Accepted: 21 February 2017

Published online: 23 February 2017

\section{References}

1. Siddik $\mathrm{ZH}$. Cisplatin: mode of cytotoxic action and molecular basis of resistance. Oncogene. 2003;22(47):7265-79.

2. Galluzzi L, Senovilla L, Vitale I, Michels J, Martins I, Kepp O, et al. Molecular mechanisms of cisplatin resistance. Oncogene. 2012;31(15):1869-83.

3. Gasser G, Ott I, Metzler-Nolte N. Organometallic anticancer compounds. J Med Chem. 2010:54(1):3-25.

4. Federici C, Petrucci F, Caimi S, Cesolini A, Logozzi M, Borghi M, et al. Exosome release and low $\mathrm{pH}$ belong to a framework of resistance of human melanoma cells to cisplatin. PLoS One. 2014;9(2), e88193.

5. Dilruba S, Kalayda GV. Platinum-based drugs: past, present and future. Cancer Chemother Pharmacol. 2016;77(6):1103-24.

6. Herrera JM, Mendes F, Gama S, Santos I, Navarro Ranninger C, Cabrera S, et al. Design and biological evaluation of new platinum(II) complexes bearing ligands with DNA-targeting ability. Inorg Chem. 2014;53(23):12627-34.

7. Cetraz M, Sen V, Schoch S, Streule K, Golubev V, Hartwig A, et al. Platinum(IV)nitroxyl complexes as possible candidates to circumvent cisplatin resistance in RT112 bladder cancer cells. Arch Toxicol. 2017;91(2):785-97.

8. Ma J, Wang Q, Yang X, Hao W, Huang Z, Zhang J, et al. Glycosylated platinum(iv) prodrugs demonstrated significant therapeutic efficacy in cancer cells and minimized side-effects. Dalton Trans. 2016;45(29):11830-8.

9. Chang JY, Hsieh HP, Chang CY, Hsu KS, Chiang YF, Chen CM, et al. 7-Aroylaminoindoline-1-sulfonamides as a novel class of potent antitubulin agents. J Med Chem. 2006;49(23):6656-9.

10. Liu Z, Zhou Z, Tian W, Fan X, Xue D, Yu L, et al. Discovery of novel 2-N-arylsubstituted benzenesulfonamidoacetamides: orally bioavailable tubulin polymerization inhibitors with marked antitumor activities. ChemMedChem. 2012;7(4):680-93.

11. Del Solar V, Quinones-Lombrana A, Cabrera S, Padron JM, Rios-Luci C, Alvarez-Valdes A, et al. Expanding the synthesis of new trans-sulfonamide platinum complexes: cytotoxicity, SAR, fluorescent cell assays and stability studies. J Inorg Biochem. 2013;127:128-40.

12. Cossa G, Gatti L, Zunino F, Perego P. Strategies to improve the efficacy of platinum compounds. Curr Med Chem. 2009;16(19):2355-65.

13. Lovejoy KS, Serova M, Bieche I, Emami S, D'Incalci M, Broggini M, et al. Spectrum of cellular responses to pyriplatin, a monofunctional cationic antineoplastic platinum(II) compound, in human cancer cells. Mol Cancer Ther. 2011;10(9):1709-19.

14. Perez C, Diaz-Garcia CV, Agudo-Lopez A, Del Solar V, Cabrera S, AgulloOrtuno MT, et al. Evaluation of novel trans-sulfonamide platinum complexes against tumor cell lines. Eur J Med Chem. 2014;76:360-8. doi:10.1016/j. ejmech.2014.02.022.

15. Godoy LC, Anderson CT, Chowdhury R, Trudel LJ, Wogan GN. Endogenously produced nitric oxide mitigates sensitivity of melanoma cells to cisplatin. Proc Natl Acad Sci U S A. 2012;109(50):20373-8.

16. Alrwas A, Papadopoulos NE, Cain S, Patel SP, Kim KB, Deburr TL, et al. Phase I trial of biochemotherapy with cisplatin, temozolomide, and dose escalation of nab-paclitaxel combined with interleukin-2 and interferon-alpha in patients with metastatic melanoma. Melanoma Res. 2014;24(4):342-8.

17. O'Connor PM, Jackman J, Bae I, Myers TG, Fan S, Mutoh M, et al. Characterization of the p53 tumor suppressor pathway in cell lines of the National Cancer Institute anticancer drug screen and correlations with the growth-inhibitory potency of 123 anticancer agents. Cancer Res. 1997;57(19):4285-300.

18. Martinez-Rivera M, Siddik ZH. Resistance and gain-of-resistance phenotypes in cancers harboring wild-type p53. Biochem Pharmacol. 2012;83(8):1049-62.

19. Chou TC. Drug combination studies and their synergy quantification using the Chou-Talalay method. Cancer Res. 2010;70(2):440-6.

20. He G, Kuang J, Khokhar AR, Siddik ZH. The impact of S- and G2-checkpoint response on the fidelity of G1-arrest by cisplatin and its comparison to a non-cross-resistant platinum(IV) analog. Gynecol Oncol. 2011;122(2):402-9.

21. Park CM, Park MJ, Kwak HJ, Moon SI, Yoo DH, Lee HC, et al. Induction of p53-mediated apoptosis and recovery of chemosensitivity through p53 transduction in human glioblastoma cells by cisplatin. Int J Oncol. 2006;28(1):119-25 
22. Florea AM, Busselberg D. Cisplatin as an anti-tumor drug: cellular mechanisms of activity, drug resistance and induced side effects. Cancers. 2011;3(1):1351-71.

23. Collins I, Garrett MD. Targeting the cell division cycle in cancer: CDK and cell cycle checkpoint kinase inhibitors. Curr Opin Pharmacol. 2005;5(4):366-73.

24. Besson A, Dowdy SF, Roberts JM. CDK inhibitors: cell cycle regulators and beyond. Dev Cell. 2008;14(2):159-69.

25. Gartel AL, Tyner AL. The role of the cyclin-dependent kinase inhibitor p21 in apoptosis. Mol Cancer Ther. 2002;1(8):639-49.

26. Hoeferlin LA, Oleinik NV, Krupenko NI, Krupenko SA. Activation of p21Dependent G1/G2 arrest in the absence of DNA damage as an antiapoptotic response to metabolic stress. Genes Cancer. 2011;2(9):889-99.

27. Lu M, Breyssens H, Salter V, Zhong S, Hu Y, Baer C, et al. Restoring p53 function in human melanoma cells by inhibiting MDM2 and cyclin B1/CDK1phosphorylated nuclear iASPP. Cancer Cell. 2013:23(5):618-33.

28. Lavecchia A, Di Giovanni C, Novellino E. CDC25 phosphatase inhibitors: an update. Mini Rev Med Chem. 2012;12(1):62-73.

29. Romanov VS, Pospelov VA, Pospelova TV. Cyclin-dependent kinase inhibitor p21(Waf1): contemporary view on its role in senescence and oncogenesis. Biochemistry (Mosc). 2012;77(6):575-84.

30. Abbas T, Dutta A. p21 in cancer: intricate networks and multiple activities. Nat Rev Cancer. 2009;9(6):400-14.

31. Coqueret $\mathrm{O}$. New roles for $\mathrm{p} 21$ and $\mathrm{p} 27$ cell-cycle inhibitors: a function for each cell compartment? Trends Cell Biol. 2003;13(2):65-70.

32. Abella N, Brun S, Calvo M, Tapia O, Weber JD, Berciano MT, et al. Nucleolar disruption ensures nuclear accumulation of p21 upon DNA damage. Traffic. 2010;11(6):743-55.

33. Asada M, Yamada T, Ichijo H, Delia D, Miyazono K, Fukumuro K, et al. Apoptosis inhibitory activity of cytoplasmic p21(Cip1/WAF1) in monocytic differentiation. Embo J. 1999;18(5):1223-34.

34. Suzuki A, Tsutomi Y, Akahane K, Araki T, Miura M. Resistance to Fas-mediated apoptosis: activation of caspase 3 is regulated by cell cycle regulator p21WAF1 and IAP gene family ILP. Oncogene. 1998;17(8):931-9.

35. Bales ES, Dietrich C, Bandyopadhyay D, Schwahn DJ, Xu W, Didenko V, et al. High levels of expression of p27KIP1 and cyclin E in invasive primary malignant melanomas. J Invest Dermatol. 1999;113(6):1039-46.

36. Xia W, Chen JS, Zhou X, Sun PR, Lee DF, Liao Y, et al. Phosphorylation/ cytoplasmic localization of p21Cip1/WAF1 is associated with HER2/neu overexpression and provides a novel combination predictor for poor prognosis in breast cancer patients. Clin Cancer Res. 2004;10(11):3815-24.

37. Slingerland J, Pagano M. Regulation of the cdk inhibitor p27 and its deregulation in cancer. J Cell Physiol. 2000;183(1):10-7.

38. Winters ZE, Leek RD, Bradburn MJ, Norbury CJ, Harris AL. Cytoplasmic p21WAF1/CIP1 expression is correlated with HER-2/neu in breast cancer and is an independent predictor of prognosis. Breast Cancer Res. 2003;5(6):R242-9.

39. Koster R, di Pietro A, Timmer-Bosscha H, Gibcus JH, van den Berg A, Suurmeijer AJ, et al. Cytoplasmic p21 expression levels determine cisplatin resistance in human testicular cancer. J Clin Invest. 2010;120(10):3594-605.

40. Xia X, Ma Q, Li X, Ji T, Chen P, Xu H, et al. Cytoplasmic p21 is a potential predictor for cisplatin sensitivity in ovarian cancer. BMC Cancer. 2011;11:399.

\section{Submit your next manuscript to BioMed Central and we will help you at every step:}

- We accept pre-submission inquiries

- Our selector tool helps you to find the most relevant journal

- We provide round the clock customer support

- Convenient online submission

- Thorough peer review

- Inclusion in PubMed and all major indexing services

- Maximum visibility for your research

Submit your manuscript at www.biomedcentral.com/submit

Biomed Central 